\title{
Cesàro summation for random fields
}

\author{
Allan Gut \\ Uppsala University \\ Ulrich Stadtmüller \\ University of Ulm
}

\begin{abstract}
Various methods of summation for divergent series of real numbers have been generalized to analogous results for sums of i.i.d. random variables. The natural extension of results corresponding to Cesàro summation amounts to proving almost sure convergence of the Cesàro means. In the present paper we extend such results as well as weak laws and results on complete convergence to random fields, more specifically to random variables indexed by $\mathbb{Z}_{+}^{2}$, the positive two-dimensional integer lattice points.
\end{abstract}

\section{Introduction}

Various methods of summation for divergent series have been studied in the literature; see e.g. [10, 21]. Several analogous results have been proved for sums of independent, identically distributed (i.i.d.) random variables.

The most commonly studied method is Cesàro summation, which is defined as follows: Let $\left\{x_{n}, n \geq 0\right\}$ be a sequence of real numbers and set, for $\alpha>-1$,

$$
A_{n}^{\alpha}=\frac{(\alpha+1)(\alpha+2) \cdots(\alpha+n)}{n !}, \quad n=1,2, \ldots, \quad \text { and } \quad A_{0}^{\alpha}=1 .
$$

The sequence $\left\{x_{n}, n \geq 0\right\}$ is said to be $(C, \alpha)$-summable iff

$$
\frac{1}{A_{n}^{\alpha}} \sum_{k=0}^{n} A_{n-k}^{\alpha-1} x_{k} \quad \text { converges as } \quad n \rightarrow \infty .
$$

It is easily checked (with $A_{n}^{-1}=0$ for $n \geq 1$ and $A_{0}^{-1}=1$ ) that $(C, 0)$-convergence is the same as ordinary convergence, and that $(C, 1)$-convergence is the same as convergence of the arithmetic means.

Now, let $\left\{X_{k}, k \geq 1\right\}$ be i.i.d. random variables with partial sums $\left\{S_{n}, n \geq 1\right\}$, and let $X$ be a generic random variable. The following result is a natural probabilistic analog of (1.2).

Theorem 1.1 Let $0<\alpha \leq 1$. The sequence $\left\{X_{k}, k \geq 1\right\}$ is almost surely (a.s.) $(C, \alpha)$-summable iff $E|X|^{1 / \alpha}<\infty$. More precisely,

$$
\frac{1}{A_{n}^{\alpha}} \sum_{k=0}^{n} A_{n-k}^{\alpha-1} X_{k} \stackrel{a . s .}{\rightarrow} \mu \quad \text { as } n \rightarrow \infty \quad \Longleftrightarrow E|X|^{1 / \alpha}<\infty \text { and } E X=\mu .
$$

For $\alpha=1$ this is, of course, the classical Kolmogorov strong law. For proofs we refer to [14] $\left(\frac{1}{2}<\alpha<1\right)$, [1] $\left(0<\alpha<\frac{1}{2}\right)$ and [2] $\left(\alpha=\frac{1}{2}\right)$.

AMS 2000 subject classifications. Primary 60F15, 60G50, 60G60, 40G05; Secondary 60F05.

Keywords and phrases. Cesàro summation, sums of i.i.d. random variables, complete convergence, convergence in probability, almost sure convergence, strong law of large numbers.

Abbreviated title. Cesàro summation for random fields.

Date. April 20, 2022 
Convergence in probability for strongly integrable random variables taking their values in real separable Banach spaces was establised in [11] under the assumption of strong integrability. In the real valued case finite mean is not necessary; for $\alpha=1$ we obtain Feller's weak law of large numbers for which a tail condition is both necessary and sufficient; cf. e.g. [8], Section 6.4.1.

Next we present Theorem 2.1 of [7] where complete convergence was obtained.

Theorem 1.2 Let $0<\alpha \leq 1$. The sequence $\left\{X_{k}, k \geq 1\right\}$ converges completely to $\mu$, i.e.,

$$
\sum_{n=1}^{\infty} P\left(\left|\sum_{k=0}^{n} A_{n-k}^{\alpha-1} X_{k}-\mu\right|>A_{n}^{\alpha} \varepsilon\right)<\infty \quad \text { for every } \varepsilon>0
$$

if and only if

$$
\begin{cases}E|X|^{1 / \alpha}<\infty, & \text { for } 0<\alpha<\frac{1}{2} \\ E|X|^{2} \log ^{+}|X|<\infty, & \text { for } \quad \alpha=\frac{1}{2} \\ E|X|^{2}<\infty, & \text { for } \quad \frac{1}{2}<\alpha \leq 1\end{cases}
$$

and $E X=\mu$.

Here and in the following $\log ^{+} x=\max \{\log x, 1\}$.

The aim of the present paper is to generalize these results to random fields. For simplicity we shall focus on random variables indexed by $\mathbb{Z}_{+}^{2}$, leaving the corresponding results for the index set $\mathbb{Z}_{+}^{d}, d>2$, to the readers.

The definition of Cesàro summability for arrays extends as follows:

Definition 1.1 Let $\alpha, \beta>0$. The array $\left\{x_{m, n}, m, n \geq 0\right\}$ is said to be $(C, \alpha, \beta)$-summable iff

$$
\frac{1}{A_{m}^{\alpha} A_{n}^{\beta}} \sum_{m, n} \sum_{k, l=0}^{m, n} A_{n-k}^{\alpha-1} A_{n-l}^{\beta-1} x_{k, l} \quad \text { converges as } m, n \rightarrow \infty .
$$

Our setup thus is the set $\left\{X_{k, l},(k, l) \in \mathbb{Z}_{+}^{2}\right\}$ with partial sums $S_{m, n},(m, n) \in \mathbb{Z}_{+}^{2}$. The Kolmogorov and Marcinkiewicz-Zygmund strong law runs as follows.

Theorem 1.3 Let $0<r<2$, and suppose that $X,\left\{X_{\mathbf{k}}, \mathbf{k} \in \mathbb{Z}^{d}\right\}$ are i.i.d. random variables with partial sums $S_{\mathbf{n}}=\sum_{\mathbf{k} \leq \mathbf{n}} X_{\mathbf{k}}, \mathbf{n} \in \mathbb{Z}^{d}$. If $E|X|^{r}\left(\log ^{+}|X|^{d-1}\right)<\infty$, and $E X=0$ when $1 \leq r<2$, then

$$
\frac{S_{\mathbf{n}}}{|\mathbf{n}|^{1 / r}} \stackrel{\text { a.s. }}{\rightarrow} 0 \quad \text { as } \quad \mathbf{n} \rightarrow \infty \text {. }
$$

Conversely, if almost sure convergence holds as stated, then $E|X|^{r}\left(\log ^{+}|X|^{d-1}\right)<\infty$, and $E X=0$ when $1 \leq r<2$.

Here $|\mathbf{n}|=\prod_{k=1}^{d} n_{i}$ and $\mathbf{n} \rightarrow \infty$ means $\inf _{1 \leq k \leq d} n_{i} \rightarrow \infty$, that is, all coordinates tend to infinity. The theorem was proved in [18] for the case $r=1$ and, generally, in [5].

For the analogous weak laws a finite moment of order $r$ suffices (in fact, even a little less), since convergence in probability is independent of the order of the index set.

The central object of investigation in the present paper is

$$
\frac{1}{A_{m}^{\alpha} A_{n}^{\beta}} \sum_{k, l=0}^{m, n} A_{m-k}^{\alpha-1} A_{n-l}^{\beta-1} X_{k, l},
$$

for which we shall establish conditions for convergence in probability, almost sure convergence and complete convergence

Let us already at this point observe that for $\alpha=\beta=1$ the quantity in (1.4) reduces to that of Theorem 1.3 with $r=1$, that is, to the multiindex Kolmogorov strong law obtained in [18]. A second thought leads us to extensions of Theorem 1.3 to the case when we do not normalize the 
partial sums with the product of the coordinates raised to some power, but the product of the coordinates raised to different powers, viz., to, for example $(d=2)$,

$$
\frac{S_{m, n}}{m^{\alpha} n^{\beta}} \quad \text { for } \quad 0<\alpha<\beta \leq 1,
$$

(where thus the case $\alpha=\beta=1 / r$ relates to Theorem 1.3). Here we only mention that some surprises occur depending on the domain of the parameters $\alpha$ and $\beta$. For details concerning this "asymmetric" Kolmogorov-Marcinkiewicz-Zygmund extension we refer to 9 .

After some preliminaries we present our results for the different modes of convergence mentioned above. A final appendix contains a collection of so-called elementary but tedious calculations.

\section{Preliminaries}

Here we collect some facts that will be used on and off, in general without specific reference.

- The first fact we shall use is that whenever weak forms of convergence or sums of probabilites are inyvolved we may equivalently compute sums "backwards", which, in view of the i.i.d. assumption shows that, for example

$$
\sum_{m, n} \sum_{k, l=0}^{m, n} P\left(A_{m-k}^{\alpha-1} A_{n-l}^{\beta-1}\left|X_{k}\right|>A_{m}^{\alpha} A_{n}^{\beta}\right)<\infty \Longleftrightarrow \sum_{m, n} \sum_{k, l=1}^{m, n} P\left(A_{k}^{\alpha-1} A_{l}^{\beta-1}|X|>A_{m}^{\alpha} A_{n}^{\beta}\right)<\infty
$$

In the same vein the order of the index set is irrelevant, that is, one-dimensional results and methods remain valid.

- Secondly we recall from (1.1) that $A_{0}^{\alpha}=1$ and that.

$$
A_{n}^{\alpha}=\frac{(\alpha+1)(\alpha+2) \cdots(\alpha+n)}{n !}, \quad n=1,2, \ldots,
$$

which behaves asymptotically as

$$
A_{n}^{\alpha} \sim \frac{n^{\alpha}}{\Gamma(\alpha+1)} \quad \text { as } n \rightarrow \infty
$$

where $\sim$ denotes that the limit as $n \rightarrow \infty$ of the ratio between the members on either side equals 1. Combining the two relations above tells us that

$$
\sum_{m, n} \sum_{k, l=0}^{m, n} P\left(A_{m-k}^{\alpha-1} A_{n-l}^{\beta-1}|X|>A_{m}^{\alpha} A_{n}^{\beta}\right)<\infty \quad \Longleftrightarrow \sum_{m, n} \sum_{k, l=1}^{m, n} P\left(k^{\alpha-1} l^{\beta-1}|X|>m^{\alpha} n^{\beta}\right)<\infty
$$

- We shall also make abundant use of the fact that if $\left\{a_{k} \in \mathbb{R}, k \geq 1\right\}$, then

$$
a_{n} \rightarrow 0 \quad \text { as } \quad n \rightarrow \infty \quad \Longrightarrow \quad \frac{1}{n} \sum_{k=1}^{n} a_{k} \rightarrow 0 \quad \text { as } \quad n \rightarrow \infty
$$

that if, in addition, $w_{k} \in \mathbb{R}^{+}, k \geq 1$, with $B_{n}=\sum_{k=1}^{n} w_{k}, n \geq 1$, where $B_{n} \nearrow \infty$ as $n \rightarrow \infty$, then

$$
\frac{1}{B_{n}} \sum_{k=1}^{n} w_{k} a_{k} \rightarrow 0 \quad \text { as } \quad n \rightarrow \infty,
$$

as well as integral versions of the same. 


\section{Convergence in probability}

We thus begin by investigating convergence in probability. We do not aim at optimal conditions, except that, as will be seen, the weak law does not require finiteness of the mean (whereas the strong law does so).

Theorem 3.1 Let $0<\alpha \leq \beta \leq 1$ and suppose that $\left\{X_{k, l}, k, l \geq 0\right\}$ are i.i.d. random variables. Further, set, for $0 \leq k \leq m, 0 \leq l \leq n$,

$$
Y_{k, l}^{m, n}=A_{m-k}^{\alpha-1} A_{n-l}^{\beta-1} X_{k, l} I\left\{\left|X_{k, l}\right| \leq A_{m}^{\alpha} A_{n}^{\beta}\right\}, \quad S_{m, n}^{\prime}=\sum_{k, l=0}^{m, n} Y_{k, l}^{m, n} \quad \text { and } \quad \mu_{m, n}=E S_{m, n}^{\prime} .
$$

Then

$$
\frac{1}{A_{m}^{\alpha} A_{n}^{\beta}}\left(\sum_{k, l=0}^{m, n} A_{m-k}^{\alpha-1} A_{n-l}^{\beta-1} X_{k, l}-\mu_{m, n}\right) \stackrel{p}{\rightarrow} 0 \quad \text { as } \quad m, n \rightarrow \infty
$$

if

$$
n P(|X|>n) \rightarrow 0 \quad \text { as } \quad n \rightarrow \infty
$$

If, in addition,

$$
\frac{\mu_{m, n}}{A_{m}^{\alpha} A_{n}^{\beta}} \rightarrow 0 \quad \text { as } \quad m, n \rightarrow \infty
$$

then

$$
\frac{1}{A_{m}^{\alpha} A_{n}^{\beta}} \sum_{k, l=0}^{m, n} A_{m-k}^{\alpha-1} A_{n-l}^{\beta-1} X_{k, l} \stackrel{p}{\rightarrow} 0 \quad \text { as } \quad m, n \rightarrow \infty .
$$

REMARK 3.1 Condition (3.2) is short of $E|X|<\infty$, i.e., the theorem extends the KolmogorovFeller weak law [12, [13, and [3], Section VII.7, to a weak law for weigthed random fields for a class of weights decaying as powers of order less than 1 in each direction.

Corollary 3.1 If, in addition, $E X=0$, then (3.4) holds (and if the mean $\mu$ is not equal to zero the limit in (3.4) equals $\mu$ ).

Corollary 3.2 If, in addition, the distribution of the summands is symmetric, then (3.2) alone suffices for (3.4) to hold.

Proof of TheOrem 3.1. The proof of the theorem amounts to an application of the so-called degenerate convergence criterion, see e.g. 8], Theorem 6.3.3.

Recalling (2.1) and (2.3) we may, equivalently, prove the theorem for the respective powers of $k$ and $l$, viz., we redefine the truncated means as

$$
Y_{k, l}^{m, n}=k^{\alpha-1} l^{\beta-1} X_{k, l} I\left\{k^{\alpha-1} l^{\beta-1}\left|X_{k, l}\right| \leq m^{\alpha} n^{\beta}\right\},
$$

with partial sums and means as

$$
S_{m, n}^{\prime}=\sum_{k, l=1}^{m, n} Y_{k, l}^{m, n} \quad \text { and } \quad \mu_{m, n}=E S_{m, n}^{\prime}
$$

respectively.

In order to check the conditions of the degenerate convergence criterion we thus wish to show that, if 3.2 is satisfied, then

$$
\sum_{k, l=1}^{m, n} P\left(k^{\alpha-1} l^{\beta-1}|X|>m^{\alpha} n^{\beta}\right) \rightarrow 0 \quad \text { as } \quad m, n \rightarrow \infty
$$


and that

$$
\frac{1}{m^{\alpha} n^{\beta}} \sum_{k, l=1}^{m, n} \operatorname{Var}\left(Y_{k, l}^{m, n}\right) \rightarrow 0 \quad \text { as } \quad m, n \rightarrow \infty .
$$

As for (3.7),

$$
\sum_{k, l=1}^{m, n} P\left(k^{\alpha-1} l^{\beta-1}|X|>m^{\alpha} n^{\beta}\right)=\frac{1}{m^{\alpha} n^{\beta}} \sum_{k, l=1}^{m, n} k^{\alpha-1} l^{\beta-1} \cdot m^{\alpha} n^{\beta} k^{1-\alpha} l^{1-\beta} P\left(k^{\alpha-1} l^{\beta-1}|X|>m^{\alpha} n^{\beta}\right),
$$

which converges to 0 as $m, n \rightarrow \infty$ via (2.5).

In order to verify (3.8) we apply the usual "slicing device" to obtain

$$
\begin{aligned}
& \frac{1}{m^{2 \alpha} n^{2 \beta}} \sum_{k, l=1}^{m, n} \operatorname{Var}\left(Y_{k, l}^{m, n}\right) \leq \frac{1}{m^{2 \alpha} n^{2 \beta}} \sum_{k, l=1}^{m, n} E\left(Y_{k, l}^{m, n}\right)^{2} \\
& \quad \leq \frac{1}{m^{2 \alpha} n^{2 \beta}} \sum_{k, l=1}^{m, n} E\left(k^{2(\alpha-1)} l^{2(\beta-1)} X^{2} I\left\{k^{\alpha-1} l^{\beta-1}|X| \leq m^{\alpha} n^{\beta}\right\}\right) \\
& \quad=\frac{1}{m^{2 \alpha} n^{2 \beta}} \sum_{k, l=1}^{m, n} k^{2(\alpha-1)} l^{2(\beta-1)} \sum_{j=1}^{m n^{\beta / \alpha}} E\left(X^{2} I\left\{(j-1)^{\alpha}<k^{\alpha-1} l^{\beta-1}|X| \leq j^{\alpha}\right\}\right) \\
& \leq \frac{1}{m^{2 \alpha} n^{2 \beta}} \sum_{k, l=1}^{m, n} \sum_{j=1}^{m n^{\beta / \alpha}} j^{2 \alpha} P\left((j-1)^{\alpha}<k^{\alpha-1} l^{\beta-1}|X| \leq j^{\alpha}\right) \\
& \quad \leq \frac{C}{m^{2 \alpha} n^{2 \beta}} \sum_{k, l=1}^{m, n} \sum_{j=1}^{m n^{\beta / \alpha}}\left(\sum_{i=1}^{j} i^{2 \alpha-1}\right) P\left((j-1)^{\alpha}<k^{\alpha-1} l^{\beta-1}|X| \leq j^{\alpha}\right) \\
& \leq \frac{C}{m^{2 \alpha} n^{2 \beta}} \sum_{k, l=1}^{m, n} \sum_{i=1}^{m n^{\beta / \alpha}} i^{2 \alpha-1} P\left(|X| \geq i^{\alpha} k^{1-\alpha} l^{1-\beta}\right) \\
&=\frac{C}{m^{\alpha} n^{\beta}} \sum_{k, l=1}^{m, n} k^{\alpha-1} l^{\beta-1}\left(\frac{1}{m^{\alpha} n^{\beta}} \sum_{i=1}^{m n^{\beta / \alpha}} i^{\alpha-1}\left(i^{\alpha} k^{1-\alpha} l^{1-\beta} P\left(|X| \geq i^{\alpha} k^{1-\alpha} l^{1-\beta}\right)\right)\right) \\
& \rightarrow 0 \quad \text { as } m, n \rightarrow \infty
\end{aligned}
$$

by applying (2.5) twice to (3.2). This completes the proof of (3.1), from which (3.4) is immediate.

Proof of Corollary 3.1. In order to conclude that also (3.4) holds we use the usual method to show that the normalized trruncated means tend to zero, where w.l.o.g. we assume that $E X=0$. Then

$$
\begin{aligned}
& \left|\frac{1}{m^{\alpha} n^{\beta}} \sum_{k, l=1}^{m, n} E\left(k^{(\alpha-1)} l^{(\beta-1)} X I\left\{k^{(\alpha-1)} l^{(\beta-1)}|X| \leq m^{\alpha} n^{\beta}\right\}\right)\right| \\
& \quad=\left|-\frac{1}{m^{\alpha} n^{\beta}} \sum_{k, l=1}^{m, n} E\left(k^{(\alpha-1)} l^{(\beta-1)} X I\left\{k^{(\alpha-1)} l^{(\beta-1)}|X|>m^{\alpha} n^{\beta}\right\}\right)\right| \\
& \quad \leq \frac{1}{m^{\alpha} n^{\beta}} \sum_{k, l=1}^{m, n} E\left(k^{(\alpha-1)} l^{(\beta-1)}|X| I\left\{k^{(\alpha-1)} l^{(\beta-1)}|X|>m^{\alpha} n^{\beta}\right\}\right) \rightarrow 0 \quad \text { as } n, m \rightarrow \infty .
\end{aligned}
$$

Proof of Corollary 3.2, Immediate, since the truncated means are (also) equal to zero. 


\section{Complete convergence}

Theorem 4.1 Let $0<\alpha \leq \beta \leq 1$. The field $\left\{X_{k, l}, k, l \geq 0\right\}$ converges completely to $\mu$, i.e.,

$$
\sum_{m n} P\left(\left|\sum_{k, l=0}^{m, n} A_{m-k}^{\alpha-1} A_{n-l}^{\beta-1} X_{k, l}-\mu\right|>A_{m}^{\alpha} A_{n}^{\beta} \varepsilon\right)<\infty \quad \text { for every } \varepsilon>0,
$$

if and only if

$$
\begin{cases}E|X|^{\frac{1}{\alpha}}, & \text { for } \quad 0<\alpha<1 / 2, \alpha<\beta \leq 1, \\ E|X|^{\frac{1}{\alpha}} \log ^{+}|X|, & \text { for } \quad 0<\alpha=\beta<\frac{1}{2}, \\ E|X|^{2}\left(\log ^{+}|X|\right)^{3}, & \text { for } \quad \alpha=\beta=\frac{1}{2}, \\ E|X|^{2}\left(\log ^{+}|X|\right)^{2}, & \text { for } \quad \alpha=\frac{1}{2}<\beta \leq 1, \\ E|X|^{2} \log ^{+}|X|, & \text { for } \quad \frac{1}{2}<\alpha \leq \beta \leq 1 .\end{cases}
$$

and $E X=\mu$.

Proof. For the proof of the sufficiency we refer to the Appendix.

As for the necessity, we argue as in [6, p. 59. We first suppose that the distribution is symmetric. Now, if complete convergence holds, then, using the fact that

$$
\max _{0 \leq k, l \leq m, n} A_{m-k}^{\alpha-1} A_{n-l}^{\beta-1}\left|X_{k, l}\right| \leq 2 \max _{0 \leq \mu, \nu \leq m, n}\left|\sum_{k, l=0}^{\mu, \nu} A_{m-k}^{\alpha-1} A_{n-l}^{\beta-1} X_{k, l}\right|,
$$

together with the Lévy inequalities we must have, say,

$$
\sum_{m, n} P\left(\max _{0 \leq k, l \leq m, n} A_{m-k}^{\alpha-1} A_{n-l}^{\beta-1}\left|X_{k, l}\right|>A_{m}^{\alpha} A_{n}^{\beta}\right)<\infty,
$$

so that, by the first Borel-Cantelli lemma

$$
P\left(A_{m-k}^{\alpha-1} A_{n-l}^{\beta-1}\left|X_{k, l}\right|>A_{m}^{\alpha} A_{n}^{\beta} \quad \text { i.o. for } 1 \leq k, l \leq m, n ; m, n \geq 1\right)=0 .
$$

At this point we use a device from [17, p. 379. Namely, if the sums $\sum_{k, l=1}^{m, n} A_{m-k}^{\alpha-1} A_{n-l}^{\beta-1} X_{k, l}$ were independent, we would conclude that $\sum_{m, n} \sum_{k, l=1}^{m, n} P\left(A_{m-k}^{\alpha-1} A_{n-l}^{\beta-1}|X|>A_{m}^{\alpha} A_{n}^{\beta}\right)$ were finite. Since, however, finiteness of the sum is only a matter of the tail probabilities, the sum is also finite in the general case.

An application of (A.6) now tells us that the finiteness of the sum is equivalent to the moment conditions as given in the statement of the theorem.

This proves the necessity in the symmetric case. The general case follows the standard desymmetrization procedure, for which we use Theorem 3.1 in order to take care of the asymptotics for the normalized medians (cf. [5, p. 472 for analogous details in the multiindex setting of the Marcinkiewicz-Zygmund strong laws).

\section{Almost sure convergence}

Theorem 5.1 Let $0<\alpha \leq \beta \leq 1$. The field $\left\{X_{k, l}, k, l \geq 0\right\}$ is almost surely (a.s.) $(C, \alpha, \beta)$ summable, that is,

$$
\frac{1}{A_{m}^{\alpha} A_{n}^{\beta}} \sum_{k, l=0}^{m, n} A_{m-k}^{\alpha-1} A_{n-l}^{\beta-1} X_{k, l} \stackrel{\text { a.s. }}{\rightarrow} \mu \quad \text { as } \quad n, m \rightarrow \infty
$$


if and only if

$$
\left\{\begin{array}{lll}
E|X|^{\frac{1}{\alpha}}, & \text { for } & 0<\alpha<\beta \leq 1 \\
E|X|^{\frac{1}{\alpha}} \log ^{+}|X|, & \text { for } & 0<\alpha=\beta \leq 1 .
\end{array}\right.
$$

and $E X=\mu$.

Proof. Since complete convergence always implies almost sure convergence, the sufficiency follows immediately for the case $\alpha<1 / 2$.

Thus, let in the following $1 / 2 \leq \alpha \leq \beta \leq 1$. We first consider the symmetric case (and recall Section 2, In analogy with [11, p. 538, the moment assumptions permit us to choose an array $\left\{\eta_{k, l}, k, l \geq 1\right\}$ of nonincreasing reals in $(0,1)$ converging to 0 , and such that

$$
\sum_{k, l=1}^{\infty} P\left(\left|X_{k, l}\right|>\eta_{k, l} k^{\alpha} l^{\beta}\right)<\infty .
$$

Defining

$$
Y_{k, l}=X_{k, l} I\left\{\left|X_{k, l}\right| \leq \eta_{k, l} k^{\alpha} l^{\beta}\right\} \quad \text { and } \quad S_{m, n}^{\prime}=\sum_{k, l=0}^{m, n} Y_{k, l}^{m, n},
$$

it thus remains to prove the theorem for the truncated sequence.

This will be achieved via the multiindex Kolmogorov convergence criterion (see e.g [4) and the multiindex Kronecker lemma (cf. [16]). The first series has just been taken care of, the second one vanishes since we are in the symmetric case, so it remains to check the third series.

Toward that end, let, for $k, l \geq 1$,

$$
Z_{k, l}=\frac{(m-k)^{\alpha-1}(n-l)^{\beta-1}}{m^{\alpha} n^{\beta}} Y_{k, l} .
$$

Then

$$
\left|Z_{k, l}\right| \leq \frac{(m-k)^{\alpha-1}(n-l)^{\beta-1}}{m^{\alpha} n^{\beta}} k^{\alpha} l^{\beta} \eta_{k, l} \leq \eta_{k, l} \leq \eta_{00} .
$$

Now, for any $\varepsilon>0$, arbitrarily small, we may choose our $\eta$-sequence such that $\eta_{00}<\varepsilon$, so that an application of the (iterated) Kahane-Hoffman-Jørgensen inequality (cf. 8], Theorem 3.7.5) yields

$$
\begin{aligned}
P\left(\left|\sum_{k, l=0}^{m, n} Z_{k, l}\right|>3^{j} \varepsilon\right) & \leq C_{j}\left(P\left(\left|\sum_{k, l=0}^{m, n} Z_{k, l}\right|>\varepsilon\right)\right)^{2^{j}} \\
& \leq C_{j}\left(\frac{\sum_{k, l=0}^{m, n}\left((m-k)^{(\alpha-1)}(n-l)^{\beta-1}\right)^{1 / \alpha} E|X|^{1 / \alpha}}{\left(\varepsilon m^{\alpha} n^{\beta}\right)^{1 / \alpha}}\right)^{2^{j}} \\
& =C_{j}^{\prime}\left(\frac{\sum_{k, l=0}^{m, n} k^{(1-1 / \alpha)} l^{(\beta-1) / \alpha}}{m n^{\beta / \alpha}}\right)^{2^{j}} \\
& = \begin{cases}C_{j}^{\prime \prime}\left(\frac{1}{(m n)^{\frac{1}{\alpha}-1}}\right)^{2^{j}}, & \text { for } \frac{1}{2}<\alpha<\beta<1, \\
C_{j}^{\prime \prime}\left(\frac{\log m}{n m}\right)^{2^{j}}, & \text { for } \frac{1}{2}=\alpha<\beta<1, \\
C_{j}^{\prime \prime}\left(\frac{\log m \log n}{n m}\right)^{2^{j}}, & \text { for } \frac{1}{2}=\alpha=\beta,\end{cases}
\end{aligned}
$$

(since the usual first term in the RHS vanishes in view of (5.1)).

By choosing $j$ sufficiently large it then follows that

$$
\sum_{m, n} \sum_{k, l=0}^{m, n} P\left(\left|\sum_{k, l=0}^{m, n} Z_{k, l}\right|>3^{j} \varepsilon\right)<\infty .
$$


By replacing $3^{j} \varepsilon$ by $\varepsilon$ we have thus, due to the arbitrariness of $\varepsilon$, shown that

$$
P\left(\left|Z_{k, l}\right|>\varepsilon \text { i.o. }\right)=0 \quad \text { for any } \varepsilon>0,
$$

from which the desired almost sure convergence follows via the multiindex Kronecker lemma referred to above.

This proves the sufficiency in the symmetric case from which the general case follows by the standard desymmetrization procedure hinted at in the proof of Theorem 4.1 .

Finally, suppose that almost sure convergence holds as stated. It then follows that

$$
\frac{A_{0}^{\alpha-1} A_{0}^{\beta-1} X_{m, n}}{A_{m}^{\alpha} A_{n}^{\beta}} \stackrel{\text { a.s. }}{\rightarrow} 0 \quad \text { as } \quad m, n \rightarrow \infty
$$

and, hence, also that

$$
\frac{X_{m, n}}{m^{\alpha} n^{\beta}} \stackrel{a . s .}{\rightarrow} 0 \quad \text { as } \quad m, n \rightarrow \infty,
$$

which, in view of i.i.d. assumption and the second Borel-Cantelli lemma, tells us that

$$
\sum_{m, n} P\left(|X|>m^{\alpha} n^{\beta}\right)<\infty
$$

which, in turn, is equivalent to the given moment conditions.

This concludes the proof of the theorem.

\section{Concluding remarks}

We close with some comments on the present and related work.

- Convergence in probability has earlier been established in 11 via approximation with indicator variables, and under the assumption of finite mean. Our proof is simpler (more elementary) and presupposes only a Feller condition.

- As pointed out above, almost sure convergence was established in three steps (14, [1] and [2]) with different proofs. Our proof, which also works for the case $d=1$, takes care of the whole proof in one go (since our proof also works for the case $\alpha<1 / 2$ ).

- For simplicity we have confined ourselves to the case $d=2$. The same ideas can be modified for the case $d>2$ and $\left(C, \alpha_{1}, \alpha_{2}, \ldots, \alpha_{d}\right)$-summability. However, the moment conditions then depend on the number of $\alpha$ :s that are equal to the smallest one (corresponding to $\alpha<\beta$ or $\alpha=\beta$ in the present paper); see [9] for Kolmogorov-Marcinkiewicz-Zygmund laws.

- Results on complete convergence are special cases of results on convergence rates. In this vein our results are extendable to results concerning

$$
\sum_{m, n} n^{r-2} m^{r-2} P\left(\left|\sum_{k, l=0}^{m, n} A_{m-k}^{\alpha-1} A_{n-l}^{\beta-1} X_{k, l}-\mu\right|>A_{m}^{\alpha} A_{n}^{\beta} \varepsilon\right)<\infty \quad \text { for every } \varepsilon>0
$$

(cf. 7] for the case $d=1$ ). For the proofs one would need i.a. extensions of the relevant computations in the appendix below.

\section{A Appendix}

In this appendix we collect a number of so-called elementary but tedious calculations. 
First, let $0<\alpha \leq \beta<1$. Then

$$
\begin{aligned}
& \sum_{m, n} \sum_{k, l=1}^{m, n} P\left(k^{\alpha-1} l^{\beta-1}|X|>m^{\alpha} n^{\beta}\right)<\infty \Longleftrightarrow \\
& \int_{1}^{\infty} \int_{1}^{\infty} \int_{1}^{x} \int_{1}^{y} P\left(|X|>u^{1-\alpha} v^{1-\beta} x^{\alpha} y^{\beta}\right) d u d v d x d y<\infty \Longleftrightarrow \\
& {\left[u^{1-\alpha} x^{\alpha}=z, \quad v^{1-\beta} y^{\beta}=w\right]} \\
& \int_{1}^{\infty} \int_{1}^{\infty} \int_{x^{\alpha}}^{x} \int_{y^{\beta}}^{y}\left(\frac{z}{x}\right)^{\frac{\alpha}{1-\alpha}}\left(\frac{w}{y}\right)^{\frac{\beta}{1-\beta}} P(|X|>z w) d z d w d x d y<\infty \\
& \int_{1}^{\infty} \int_{1}^{\infty}\left(\int_{z}^{z^{1 / \alpha}} \frac{d x}{x^{\frac{\alpha}{1-\alpha}}}\right)\left(\int_{w}^{w^{1 / \beta}} \frac{d y}{y^{\frac{\beta}{1-\beta}}}\right) z^{\frac{\alpha}{1-\alpha}} w^{\frac{\beta}{1-\beta}} P(|X|>z w) d z d w<\infty .
\end{aligned}
$$

In case $0<\alpha<\beta=1$ we have

$$
\begin{aligned}
& \sum_{m, n} \sum_{k, l=1}^{m, n} P\left(k^{\alpha-1}|X|>m^{\alpha} n\right)<\infty \Longleftrightarrow \\
& \int_{1}^{\infty} \int_{1}^{\infty}\left(\int_{z}^{z^{1 / \alpha}} \frac{d x}{x^{\frac{\alpha}{1-\alpha}}}\right) z^{\frac{\alpha}{1-\alpha}} w P(|X|>z w) d z d w<\infty .
\end{aligned}
$$

Next we note that

$$
\int_{y}^{y^{1 / \gamma}} \frac{d x}{x^{\frac{\gamma}{1-\gamma}}} \sim C \begin{cases}y^{\frac{1-2 \gamma}{\gamma(1-\gamma)}}, & \text { for } 0<\gamma<\frac{1}{2} \\ \log y, & \text { for } \gamma=\frac{1}{2} \\ y^{\frac{1-2 \gamma}{1-\gamma}}, & \text { for } \frac{1}{2}<\gamma<1\end{cases}
$$

so that

$$
\begin{aligned}
\left(\int_{z}^{z^{1 / \alpha}} \frac{d x}{x^{\frac{\alpha}{1-\alpha}}}\right) & \left(\int_{w}^{w^{1 / \beta}} \frac{d y}{y^{\frac{\beta}{1-\beta}}}\right)^{\frac{\alpha}{1-\alpha}} w^{\frac{\beta}{1-\beta}} \\
& \sim C \begin{cases}z^{\frac{1-\alpha}{\alpha}} w^{\frac{1-\beta}{\beta}}, & \text { for } \quad 0<\alpha, \beta<\frac{1}{2}, \\
(z w)^{\frac{1-\alpha}{\alpha}}, & \text { for } \quad 0<\alpha=\beta<\frac{1}{2}, \\
z w \log z \log w=\frac{z w}{2}\left((\log z w)^{2}\right. & \text { for } \quad \alpha=\beta=\frac{1}{2}, \\
\left.z^{\frac{1-\alpha}{\alpha}} w \log w,(\log z)^{2}-(\log w)^{2}\right), & \text { for } \quad \alpha<\beta=\frac{1}{2}, \\
z^{\frac{1-\alpha}{\alpha}} w, & \text { for } \quad \alpha<\frac{1}{2}<\beta \leq 1, \\
z w \log z, & \text { for } \quad \alpha=\frac{1}{2}<\beta \leq 1, \\
z w, & \text { for } \quad \frac{1}{2}<\alpha \leq \beta \leq 1,\end{cases}
\end{aligned}
$$


from which it follows that

$$
\begin{gathered}
\int_{1}^{\infty} \int_{1}^{\infty}\left(\int_{z}^{z^{1 / \alpha}} \frac{d x}{x^{\frac{\alpha}{1-\alpha}}}\right)\left(\int_{w}^{w^{1 / \beta}} \frac{d y}{y^{\frac{\beta}{1-\beta}}}\right) z^{\frac{\alpha}{1-\alpha}} x^{\frac{\beta}{1-\beta}} P(|X|>z w) d z d w \\
=[x=z w, \quad y=z] \\
= \begin{cases}\int_{1}^{\infty} \int_{1}^{x} x^{\frac{1-\beta}{\beta}} y^{\frac{1}{\alpha}-\frac{1}{\beta}-1} P(|X|>x) d y d x & \text { for } \quad 0<\alpha<\beta<\frac{1}{2}, \\
=C \int_{1}^{\infty} x^{\frac{1}{\alpha}-1} P(|X|>x) d x, & \text { for } \quad 0<\alpha=\beta<\frac{1}{2}, \\
\int_{1}^{\infty} \int_{1}^{x} x^{\frac{1-\alpha}{\alpha}} \frac{1}{y} P(|X|>x) d y d x & \text { for } \quad \alpha=\beta=\frac{1}{2}, \\
=C \int_{1}^{\infty} x^{\frac{1-\alpha}{\alpha}} \log x P(|X|>x) d x, & \text { for } \quad \alpha<\beta=\frac{1}{2}, \\
\int_{1}^{\infty} \int_{1}^{x}\left(\frac{1}{2} x(\log x)^{2} \frac{1}{y}-x \frac{(\log y)^{2}}{y}\right) P(|X|>x) d x d y & \\
=\frac{1}{6} \int_{1}^{\infty} x(\log x)^{3} P(|X|>x) d x, & \text { for } \quad \alpha<\frac{1}{2}<\beta \leq 1, \\
=C y^{\frac{1}{\alpha}-2}(\log x-\log y) P(|X|>x) d y d x & \text { for } \quad \alpha=\frac{1}{2}<\beta \leq 1, \\
\int_{1}^{\infty} \int_{1}^{x} x y^{\frac{1}{\alpha}-2} P(|X|>x) d y d x & \text { for } \quad \frac{1}{2}<\alpha \leq \beta \leq 1 . \\
=C \int_{1}^{\infty} x^{\frac{1}{\alpha}-1} P(|X|>x) d x, & \\
\int_{1}^{\infty} \int_{1}^{x} x \frac{\log y}{y} P(|X|>x) d y d x & \\
=\frac{1}{2} \int_{1}^{\infty} x(\log x)^{2} P(|X|>x) d x, & \\
\int_{1}^{\infty} \int_{1}^{x} x \frac{1}{y} P(|X|>x) d y d x & \frac{1}{2} \int_{1}^{\infty} x \log x P(|X|>x) d x,\end{cases}
\end{gathered}
$$

Summarizing this we have shown that, for $0<\alpha \leq \beta<1$,

$$
\begin{aligned}
& \sum_{m, n} \sum_{k, l=1}^{m, n} P\left(A_{k}^{\alpha-1} A_{l}^{\beta-1}|X|>A_{m}^{\alpha} A_{n}^{\beta}\right)<\infty \quad \Longleftrightarrow \\
& \left\{\begin{array}{lll}
E|X|^{\frac{1}{\alpha}}, & \text { for } \quad 0<\alpha<1 / 2, \alpha<\beta \leq 1, \\
E|X|^{\frac{1}{\alpha}} \log ^{+}|X|, & \text { for } \quad 0<\alpha=\beta<\frac{1}{2}, \\
E|X|^{2}\left(\log ^{+}|X|\right)^{3}, & \text { for } \quad \alpha=\beta=\frac{1}{2}, \\
E|X|^{2}\left(\log ^{+}|X|\right)^{2}, & \text { for } \quad \alpha=\frac{1}{2}<\beta \leq 1, \\
E|X|^{2} \log ^{+}|X|, & \text { for } \quad \frac{1}{2}<\alpha \leq \beta \leq 1 .
\end{array}\right.
\end{aligned}
$$

\section{Acknowledgement}

The work on this paper has been supported by Kungliga Vetenskapssamhället i Uppsala. Their support is gratefully acknowledged. In addition, the second author likes to thank his partner Allan Gut for the great hospitality during two wonderful and stimulating weeks at the University of Uppsala. 


\section{References}

[1] Chow, Y.S. And LaI, T.L. (1973). Limiting behavior of weighted sums of independent random variables. Ann. Probab. 1, 810-824.

[2] Déniel, Y. And Derriennic, Y. (1988). Sur la convergence presque sure, au sens de Cesàro d'ordre $\alpha, 0<\alpha<1$, de variables aléatoires indépendantes et identiquement distribuées. Probab. Th. Rel. Fields 79, 629-636.

[3] Feller, W. (1971). An Introduction to Probability Theory and Its Applications, Vol 2, 2nd ed. Wiley, New York.

[4] Gabriel J.-P. (1977). An inequality for sums of independent random variables indexed by finite dimensional filtering sets and its applications to the convergence of series. Ann. Probab. 5, 779-786.

[5] Gut, A. (1978). Marcinkiewicz laws and convergence rates in the law of large numbers for random variables with multidimensional indices. Ann. Probab. 6, 469-482.

[6] Gut, A. (1992). Complete convergence for arrays. Period. Math. Hungar. 25, 51-75.

[7] Gut, A. (1993). Complete convergence and Cesàro summation for i.i.d. random variables. Probab. Th. Rel. Fields 97, 169-178.

[8] Gut, A. (2007). Probability: A Graduate Course, Corr. 2nd printing. Springer-Verlag, New York.

[9] Gut, A. And Stadtmüller, U. (2008). An asymmetric Marcinkiewicz-Zygmund LLN for random fields. Report U.U.D.M. 2008:38, Uppsala University.

[10] Hardy, G.H. (1949). Divergent Series. Oxford University Press.

[11] Heinkel, B. (1990). An infinite-dimensional law of large numbers in Cesaro's sense. J. Theoret. Probab. 3, 533-546.

[12] Kolmogorov, A.N. (1928). Über die Summen durch den Zufall bestimmter unabhängiger Größen. Math. Ann. 99, 309-319.

[13] Kolmogorov, A.N. (1930). Bemerkungen zu meiner Arbeit "Über die Summen zufälliger Größen”. Math. Ann. 102, 484-488.

[14] Lorentz G.G. (1955). Borel and Banach properties of methods of summation. Duke Math. J. 22, 129-141.

[15] Marcinkiewicz, J. And Zygmund, A. Sur les fonctions indépendantes. Fund. Math. 29, 60-90 (1937).

[16] Moore, C.N. (1966). Summable Series and Convergence Factors. Dover, New York.

[17] Nerman, O. (1981). On the convergence of supercritical general (C-M-J) branching processes. Z. Wahrsch. verw. Gebiete 57, 365-395.

[18] Smythe, R. (1973). Strong laws of large number for $r$-dimensional arrays of random variables. Ann. Probab. 1, 164-170.

[19] Stadtmüller, U. And Thalmaier, M. (2008). Strong laws for delayed sums of random fields. Preprint, University of Ulm.

[20] Thalmaier, M. (2008): Grenzwertsätze für gewichtete Summen von Zufallsvariablen und Zufallsfeldern. Dissertation, University of Ulm. 
[21] Zygmund, A. (1968). Trigonometric Series. Cambridge University Press.

Allan Gut, Department of Mathematics, Uppsala University, Box 480,

SE-751 06 Uppsala, Sweden;

Email: allan.gut@math.uu.se

URL: http://www.math.uu.se/ ^allan

Ulrich Stadtmüller, Ulm University, Department of Number Theory and Probability Theory, D-89069 Ulm, Germany;

Email ulrich.stadtmueller@uni-ulm.de

URL: http://www.mathematik.uni-ulm.de/matheIII/members/stadtmueller/stadtmueller.html 